\title{
Tamoxifen in early-stage estrogen receptor- positive breast cancer: overview of clinical use and molecular biomarkers for patient selection
}

This article was published in the following Dove Press journal:

OncoTargets and Therapy

16 December 2010

Number of times this article has been viewed

\section{Carmen Criscitiello' \\ Debora Fumagalli' \\ Kamal S Saini \\ Sherene Loi'}

'Breast Cancer Translational Research Laboratory, Institut Jules Bordet, Université Libre de Bruxelles, Brussels; ${ }^{2}$ Breast Data Centre, Institut Jules Bordet, Brussels, Belgium
Correspondence: Sherene Loi Breast Cancer Translational Research Laboratory, Institut Jules Bordet, Boulevard de Waterloo I25, Brussels 1000, Belgium Tel +32025413457

Fax +32025413858

Email sherene.loi@bordet.be

\begin{abstract}
Tamoxifen was the first targeted anticancer agent for breast cancer patients and its effects on reduction of breast cancer events and improvement in overall survival are undisputed. Hence, it has long been considered an essential part of patient care. Recent results of several large adjuvant hormonal trials evaluating the use of aromatase inhibitors in comparison with the previous standard of five years of tamoxifen has led to a paradigm shift, ensuring the inclusion of an aromatase inhibitor as part of standard endocrine therapy for most postmenopausal women diagnosed today with estrogen receptor-positive breast cancer. However, one could argue that despite statistically significant improvements in breast cancer events, an overall survival advantage has not been clear. In this review, we discuss recent genomic and molecular data pertaining to estrogen receptor-positive breast cancer and how this knowledge may aid clinicians to prescribe adjuvant hormonal treatment in the future. A combination of gene expression and genetic aberration markers may be most useful in discerning a population that is still appropriate for adjuvant tamoxifen treatment.
\end{abstract}

Keywords: tamoxifen, aromatase inhibitors, resistance, prediction, mutation, endocrine therapy, PI3K

\section{Introduction}

Hormonal therapy is considered an essential part of the management of patients with estrogen receptor (ER)-positive breast cancer. Immunohistochemically confirmed presence of ER is the most important factor that predicts response to hormonal manipulation.

The ER is a transcription factor of the nuclear receptor family, and has two isoforms, ER $\alpha$ and ER $\beta$. Estradiol (E2) binding to ER induces a change in conformation which releases it from an inhibitory complex and induces dimerization. ${ }^{1}$ Regulatory proteins may act as either coactivators or corepressors to modulate the action of ER. Coactivator proteins increase transcriptional activity of DNA elements known as estrogen response elements, and result in cell survival and proliferation. ${ }^{2}$ ER may also interact with transcription factors including Fos/Jun and modulate the activity of cyclin D1, and thus impact transcription of genes that do not have estrogen response elements. These "genomic" mechanisms are referred to as nuclear-initiated steroid signaling.

About $5 \%-10 \%$ of endogenous cellular ER $\alpha$ is located near the cytoplasmic membrane. ${ }^{3}$ Membrane ERs can activate surface receptors (eg, EGFR, HER2, IGF-1R) or $\mathrm{G}$ protein-coupled receptors which then signal via classic transduction pathways including PI3K/Akt/mTOR and Ras/Raf/MEK/ERK. ${ }^{4}$ This results in activation of several kinases and phosphatases, generation of second messengers, and calcium flux. 
This distinct "nongenomic" signaling action occurs within seconds to minutes, and is known as membrane-initiated steroid signaling.

Endocrine therapies that either interrupt the synthesis of estrogens or interfere with estrogen-mediated signaling pathways have become an integral part of the management of hormone-dependent breast cancer. The most commonly used agent of this class is tamoxifen, a selective ER modulator. This review will focus on the current evidence, particularly for the use of tamoxifen, but also other hormonal drugs in ER-positive breast cancer. It will also discuss some recent biologic and genomic knowledge that could provide a mechanistic insight and may aid clinicians better identify those ER-positive breast cancer patients who could do well with tamoxifen treatment in the near future.

\section{Tamoxifen}

Tamoxifen is the most widely used hormonal treatment for breast cancer in both pre- and postmenopausal women. Adjuvant therapy with tamoxifen for five years resulted in a significant reduction in the annual breast cancer death rate of $34 \%$, with an absolute reduction in mortality of $9.2 \%$ at 15 years. $^{5}$

The antitumor effects of tamoxifen are thought to be due to its antiestrogenic activity, mediated by competitive inhibition of estrogen binding to ER. ${ }^{6}$ As a consequence, tamoxifen inhibits the expression of estrogen-regulated genes, including growth factors and angiogenic factors secreted by the tumor that may stimulate growth by autocrine or paracrine mechanisms. ${ }^{7}$ The net result is a block in the G1 phase of the cell cycle and a slowing of cell proliferation. Tumors may then regress because of this altered balance between cell proliferation and ongoing cell loss. Tamoxifen may also directly induce programmed cell death. ${ }^{8}$

De novo or acquired resistance may occur after treatment, limiting the effectiveness of tamoxifen in many patients. In at least some patients, the disease progresses during treatment because tumor growth can be stimulated by tamoxifen itself. ${ }^{9}$ Tamoxifen-stimulated growth explains the "withdrawal response" that occurs in some patients when the drug is stopped because of tumor progression, and it explains the lack of response to oophorectomy in premenopausal women if tamoxifen is not discontinued at the time tumor progression is observed..$^{10}$ Possible mechanisms of tamoxifen resistance include the presence of variant ER, the absence or loss of ER, altered expression of receptor-interacting proteins, and "cross-talk" amongst ER and other growth-factor signalling pathways. ${ }^{6,9,10}$
Some tumors spontaneously become hormone-independent despite the presence of ER; in others, tumors that are initially ER-positive become ER-negative over time. ${ }^{11,12}$ At least two-thirds of the tumors that become resistant to tamoxifen continue to express ER, and many of these tumors regress when second-line hormonal therapy is initiated.

\section{Aromatase inhibitors}

The long-term success of tamoxifen therapy has led to the development of other endocrine approaches. Because the major source of estrogen in postmenopausal women is aromatase-mediated conversion of circulating androstenedione to estrone in peripheral tissues, an alternate approach to the management of postmenopausal breast cancer has been through the use of aromatase inhibitors.

There are two distinct mechanisms by which aromatase inhibitors block the action of aromatase and reduce local and peripheral estrogen production in postmenopausal women. ${ }^{13}$ Type 1 inhibitors (exemestane) have androgen-like structures that bind irreversibly to the substrate complex, permanently inactivating aromatase and leading to prolonged estrogen deprivation. Type 2 inhibitors (letrozole and anastrozole) are nonsteroidal compounds that reversibly bind to the heme component of the aromatase enzyme. The clinical significance of these differences, if any, remains to be determined. ${ }^{14}$ They almost completely suppress aromatase activity (by 97\%-99\%) in postmenopausal women.

Hormone-sensitive breast cancer treated with aromatase inhibitors can also acquire endocrine resistance. In theory, an inefficient inhibition of the aromatase, either due to reduced drug levels or mutations/overexpression of the enzyme, may be implicated. Site-directed mutation can indeed produce aromatase that is resistant to inhibition by some, but not all, aromatase inhibitors. ${ }^{15,16}$ Nonetheless, clinical data show that changing the aromatase inhibitor class at disease progression (ie, from Type I to Type II, and vice versa) yields some clinical benefits, thus suggesting that inefficient inhibition of aromatase may drive some cases of disease progression. Exposure to long-term estrogen deprivation and subsequent development of acquired resistance may be accompanied by adaptive increases in ER gene expression and intercellular signalling, resulting in hypersensitivity to low estradiol levels. ${ }^{17,18}$ Furthermore, there is evidence of increased cross-talk between the various growth factor-receptor signalling pathways and ER at the time of relapse, with ER becoming activated and supersensitized by several different intracellular kinases. ${ }^{16}$ It would seem that, following failure of aromatase inhibitors, the ER 
remains an integral part of the pathway sustaining tumor proliferation.

Further treatment with antiestrogen agents after development of acquired resistance to aromatase inhibitors is generally accepted. Because mechanisms of acquired resistance to tamoxifen are thought to be similar to those developing after exposure to aromatase inhibitors, tamoxifen does not appear to be an ideal candidate to treat tumors regrowing after aromatase inhibitor exposure. In such an environment tamoxifen may mainly act as an agonist. ${ }^{16}$ However, recent results of the BIG 1-98 trial do not support a significant detrimental clinical effect of prescribing tamoxifen after letrozole, as previously hypothesized. ${ }^{19}$

\section{Fulvestrant}

The search for an antiestrogen devoid of the agonist activity of tamoxifen and which can effectively block ER activity resulted in the discovery and clinical development of fulvestrant. ${ }^{20,21}$

Fulvestrant is a pure ER antagonist, without known agonistic properties, that downregulates cellular levels of the ER in a dose-dependent manner. ${ }^{22-24}$ It binds to the ER but, due to its steroidal structure and long side chain, induces a different conformational shape with the receptor to that achieved by the nonsteroidal antiestrogen, tamoxifen. As a result, fulvestrant prevents ER dimerization and leads to the rapid degradation of the fulvestrant-ER complex, producing the loss (downregulation) of cellular ER. ${ }^{25}$ Fulvestrant, unlike tamoxifen, inhibits ER-DNA binding and produces abrogation of estrogen-sensitive gene transcription. ${ }^{26}$ In vitro, fulvestrant significantly inhibits the expression of genes such as c-myb and c-myc in cells resistant to long-term estrogen deprivation, and may therefore be an appropriate therapeutic option after progression on aromatase inhibitors. ${ }^{17}$ Unfortunately clinical development of fulvestrant has been hampered by poor oral bioavailability, with the intramuscular route of administration plagued by solubility and dosage problems, and confusing clinical results. Despite the encouraging preclinical data, clinical data showed only equivalence to aromatase inhibitors and tamoxifen. ${ }^{27}$ It currently seems that the true potential of this hormonal agent may never be fully realized.

\section{Adjuvant hormonal trials}

In general, the studies that have included aromatase inhibitors in the treatment of postmenopausal women with early breast cancer have evaluated their use in three distinct ways, ie, as front-line adjuvant therapy for five years in substitution of tamoxifen (up-fronting approach), as sequential adjuvant therapy following 2-3 years of tamoxifen for 3-2 years up to a total duration of five years (switching approach), or as additional five years after completion of five years of tamoxifen (extending approach). ${ }^{28,29}$

The ATAC trial compared five years of upfront anastrozole with five years of tamoxifen, or the combination of both. ${ }^{29,30}$ The combination arm showed no added benefit over the tamoxifen arm, and it was closed. A peak in recurrence at two years was observed in patients receiving tamoxifen, but not in those receiving anastrozole. At a median 100-month follow-up, disease-free survival was significantly improved in patients treated with anastrozole. No significant difference in overall survival was observed (see Table 1).

The BIG 1-98 trial randomized patients to either five years of tamoxifen, five years of letrozole, two years of tamoxifen followed by three years of letrozole, or two years of letrozole followed by three years of tamoxifen. An initial analysis, at a median follow-up of 25.8 months, demonstrated a significantly improved disease-free survival in the frontline letrozole arm. ${ }^{31}$ These results have also been confirmed at a more recent analysis (76-month median follow-up). However, this study did not show that either tamoxifen or

Table I Summary of currently reported adjuvant hormonal therapy trials. Overall, compared with five years of tamoxifen alone, the incorporation of an aromatase inhibitor significantly improves disease-free survival

\begin{tabular}{|c|c|c|c|c|c|c|c|c|c|}
\hline Study & Patients (N) & $\begin{array}{l}\text { Last follow-up } \\
\text { (months) }\end{array}$ & Al & Comparator & DFS HR & $P$ value & OS HR & $P$ value & REF \\
\hline \multicolumn{10}{|l|}{ Up-front adjuvant } \\
\hline ATAC & 5216 & 100 & ANA & TAM & 0.85 & 0.003 & 0.97 & 0.7 & 29 \\
\hline BIG I-98 (MA) & 4922 & 76 & LET & TAM & 0.88 & 0.03 & 0.81 & 0.08 & 31 \\
\hline TEAM & 9766 & 33 & EXE & TAM & 0.83 & 0.02 & NR & NR & 33 \\
\hline \multicolumn{10}{|l|}{ Switch adjuvant } \\
\hline IES & 4724 & 56 & EXE & TAM & 0.76 & 0.001 & 0.85 & 0.08 & 34 \\
\hline ABCSG 8/ITA/ARNO 95 & 2577 & 84 & ANA & TAM & 0.59 & 0.0001 & 0.71 & 0.04 & 35 \\
\hline \multicolumn{10}{|l|}{ Extended adjuvant } \\
\hline MA. 17 & 5187 & 30 & LET & Placebo & 0.58 & 0.001 & 0.98 & 0.853 & 36 \\
\hline
\end{tabular}

Abbreviation: MA, monotherapy arm. 
letrozole initial therapy had a significant effect on overall survival. An analysis of the two sequential treatment arms (with a median follow-up of 71 months) reported ${ }^{19}$ that none of the study outcomes was significantly improved using either sequential therapy compared with letrozole monotherapy. Trends support initial use of letrozole in patients at higher risk of relapse. Patients commenced on letrozole can be switched to tamoxifen if required. Updated results of the monotherapy comparison suggest a trend towards superior overall survival with letrozole compared with tamoxifen (see Table).

The TEAM (Tamoxifen Exemestane Adjuvant Multinational) trial compared upfront tamoxifen with upfront exemestane. An initial analysis at 2.75 years showed a nonsignificant trend in disease-free survival benefit, indicating an improved outcome with exemestane versus tamoxifen in the intent to treat population. ${ }^{32}$ Excluding patients who have never received a study drug, disease-free survival significantly improved with exemestane. At a median follow-up of 5.1 years, exemestane monotherapy and sequential therapy achieved similar diseasefree survival and overall survival (see Table). ${ }^{33}$

The IES study compared a switch to exemestane or continued tamoxifen after an initial 2-3 years of tamoxifen therapy, for a total of five years. An eight-year follow-up showed the switch significantly improved both disease-free survival and overall survival (see Table). ${ }^{34}$

A meta-analysis of three trials (ABCGS-8, ITA, ARNO) comparing five years of tamoxifen with tamoxifen followed by anastrozole showed a significantly improved disease-free survival and overall survival in patients in the switching arms. $^{35}$

The MA.17 study randomized patients who had completed 4.5-6.0 years of tamoxifen to either five years of subsequent letrozole therapy or placebo. ${ }^{36,37}$ At a median follow-up of 2.5 years, patients who continued adjuvant treatment with letrozole experienced significantly improved disease-free survival, but no change in overall survival (see Table). However, a retrospective subgroup analysis revealed a significant benefit in overall survival among patients with node-positive disease.

\section{Genomic contribution to definition of tamoxifen-sensitive breast cancer}

Current guidelines recommend the inclusion of adjuvant endocrine therapy in almost all patients whose tumors show evidence of endocrine responsiveness. ${ }^{38}$ However, the heterogeneity of breast cancer, clinical behavior, and response to endocrine treatment has long been apparent to clinicians, despite positive expression of ER and its related genes, and in the adjuvant setting up to $30 \%$ of patients with ER-positive breast cancer relapse despite the treatment due to either intrinsic or acquired resistance. ${ }^{5}$

Given the heterogeneity and complexity of breast cancer disease, it is probably unlikely that one predictive biomarker can capture and explain the differences in clinical outcome for all ER-positive tumors. For this reason, in recent years, whole genome microarray and other high-throughput technologies able to provide simultaneous characterization of thousands of genes have been extensively used in an attempt to define more accurate prognostic and predictive markers for breast cancer, and to obtain a mechanistic insight into the reasons underlying sensitivity and resistance to tamoxifen treatment.

\section{Molecular classification of breast cancer and prognostic implications}

Unsupervised hierarchic clustering analysis has identified multiple molecular subgroups within breast cancer. The most commonly cited microarray classification divides breast cancers into four main groups: the "basal-like" subtype, which is predominantly ER-negative, PR-negative, and HER2negative; the HER2-like subtype, characterized by increased expression of genes located in the HER2 amplicon; and at least two "luminal-like" subtypes, predominately ER-positive, named luminal A and B. ${ }^{39-42}$ Interestingly, these molecular phenotypes were associated with distinct clinical outcomes. When originally described, the luminal A subgroup had the highest expression of ER and ER-regulated genes and a better clinical outcome compared with the luminal B subgroup, suggesting an underlying molecular basis of the heterogeneity of clinical outcome in breast cancer. Relevant to this review article, a group of ER-positive cancers that had an unfavorable outcome even when treated with adjuvant endocrine treatment could be observed. ${ }^{40}$ These data heralded much early excitement and reinforced to clinicians and scientists alike that breast cancer subtypes were likely to need different therapeutics and that the biology of disease progression was probably unique for each subgroup. Unfortunately, despite these seminal publications, clinically implemented stratification of luminal A and B subtypes is currently rare.

Several predictive gene sets and assays have been developed over recent times to aid clinicians identify those ER-positive breast cancers that have a poor clinical outcome despite tamoxifen treatment in the hope of determining which patients may be suitable for tamoxifen, either alone or sequentially, and those with a poor prognosis who may 
require chemotherapy, aromatase inhibitors, or other targeted strategies. ${ }^{43-47}$ One of the most successful assays was developed by Genomic Health using quantitative real-time polymerase chain reaction and was derived by analyzing the results from three preliminary studies involving 447 samples from a heterogeneously treated population (chemotherapy and tamoxifen) and 250 candidate genes selected after a literature search of the most important microarray experiments relating to breast cancer prognosis. ${ }^{43}$ Of these, 21 genes were chosen, five of which were control genes; the derived multigene predictor assigned a "recurrence score" to predict low-, intermediate-, and high-risk of distant recurrence for women receiving five years of adjuvant tamoxifen ( \pm chemotherapy).

The predictor was subsequently validated prospectively in archival patients who had received tamoxifen only in the NSABP B-14 trial. The recurrence score accurately predicted patients at high-risk versus low-risk of recurrence on tamoxifen, identifying those who do poorly with tamoxifen treatment. The recurrence score is currently the subject of a global Phase III randomized trial evaluating its ability to select ER-positive breast cancer patients for either adjuvant hormonal treatment alone or with standard chemotherapy.

The gene expression grade index (GGI) score is based on 97 genes found to be consistently differentially expressed between low (Grade 1) and high (Grade 3) histologic grade breast cancer. ${ }^{49}$ Not surprisingly, these genes are mainly involved in cell cycle regulation, proliferation, apoptosis evasion, and differentiation. Of note, the GGI was shown to be able to define two distinct molecular subgroups within ERpositive breast cancer that were highly comparable with the previously defined luminal A and B classification. The samples previously classified as luminal A or B were associated with significantly different GGI values, with luminal A subtypes being associated with low GGI values and the luminal B tumors having significantly higher GGI values. Of note, these two subtypes were associated with statistically distinct outcomes in both systematically untreated and tamoxifen-treated populations. ${ }^{47,49}$ These studies were important because they reinforced the hypothesis that the proliferative phenotype is associated with a poor prognosis in ER-positive breast cancer, thus highlighting a group not suitable for tamoxifen monotherapy and a group with a critical need for further research. The molecular "drivers" of the luminal B or highly proliferative ER-positive phenotype, and what may be its best therapeutic strategy remain to be determined. It is probable that a few molecular targets will emerge, such as PIK3CA and FGFR1 amplification. As such, it is currently far from clear if aromatase inhibitors, fulvestrant, or chemotherapy can change the natural disease history of this subgroup. Future integrative genomic and deep sequencing studies may shed further biologic light for these breast cancer patients in the future. However, it is imperative that clinical trials prospectively stratify for luminal prognostic subtypes using some measure of tumor proliferation activity. Markers such as Ki67 immunohistochemistry, histologic grade, GGI, or recurrence score may be useful in this regard.

\section{Gene expression predictors of response to endocrine treatment}

While molecular profiling of breast cancer has shown the existence of different prognostic tumor phenotypes within the ER-positive population, determining if any of these gene signatures could act as a predictive biomarker of benefit from endocrine agents rather that just prognosis (ie, independent of treatment) has been more difficult. Many of the aforementioned studies involved tumors that had been treated with tamoxifen, but a poor prognosis may be related to inherent tumor aggressiveness as well as tamoxifen resistance.

The recurrence score has been reported to be predictive of tamoxifen response. In particular, a low recurrence score is considered predictive of tamoxifen benefit in ER-positive, node-negative cases, while a high recurrence score is predictive of chemotherapy benefit over hormonal therapy in ERpositive patients, regardless of lymph node status. ${ }^{48,50,51}$

In the recent past, several other gene expression signatures have been developed in an attempt to predict sensitivity or resistance to both tamoxifen and aromatase inhibitors. ${ }^{52-55}$ In a recent study, Symmans et $\mathrm{al}^{52}$ hypothesizing that measurement of gene expression related to ER within a breast cancer sample represents intrinsic tumor sensitivity to adjuvant endocrine therapy, were able to define a genomic index for sensitivity to endocrine therapy (SET index) from genes coexpressed with the ESR 1 gene in 437 microarray profiles from newly diagnosed breast cancer, unrelated to treatment or outcome. The association of the SET index and ESR1 levels with distant relapse risk was evaluated from microarrays of ER-positive breast cancer in two cohorts who received five years of tamoxifen alone as adjuvant endocrine therapy, a cohort who received neoadjuvant chemotherapy followed by tamoxifen and/or aromatase inhibitor, and two cohorts who received no adjuvant systemic therapy. The SET index (165 genes) was significantly associated with distant 
relapse or death risk in both tamoxifen-treated cohorts and in the chemoendocrine-treated cohort independent of the pathologic response to chemotherapy, but was not prognostic in two untreated cohorts. No distant relapse or death was observed after tamoxifen alone if node-negative and high SET, or after chemoendocrine therapy if intermediate or high SET. The SET index was found to be useful in estimating distant relapse-free survival if patients were to receive adjuvant endocrine therapy alone and in conjunction with other clinicopathologic information to determine whether or not additional treatment might be indicated to improve the likelihood of cure further. ${ }^{52}$

Kok et $\mathrm{al}^{45,56}$ evaluated if their 78-gene signature that was developed from a dataset of metastatic breast cancer patients who did and did not respond to tamoxifen treatment was truly predictive of tamoxifen response. They found that their signature seemed to be more predictive than prognostic compared with the recurrence score in an independent set of tamoxifen-treated ER-positive metastatic breast cancer patients. Whilst the metastatic setting may be the most logical way to investigate the true predictive ability of a biomarker, it remains plausible that metastatic breast cancer patients have different disease biology compared with those having early-stage disease.

Miller et $\mathrm{al}^{54}$ used the neoadjuvant or preoperative setting to uncover gene profiles for which baseline expression and relative change with 14 days of treatment differed between breast cancers that were clinically responsive or resistant to letrozole therapy. The advantage of the neoadjuvant setting is that it allows multiple ways of assessment of response to therapy, eg, monitoring of changes in tumor size during the first months of treatment and sequential tumor biopsies before and after neoadjuvant treatment with letrozole. Gene expression profiles were then related to clinical responses as assessed from tumor volume measurements after three months of treatment. Bioinformatic analysis identified 205 probe sets (69 baseline expression, 45 day 14 expression, and 91 changes in expression with treatment) which differentiated between clinical responders and nonresponders. Of note, the high proportion of change and day 14 variables among the total emphasizes the need to monitor the effects of therapy on gene expression as well as baseline parameters when identifying predictive markers of response to treatment. ${ }^{54}$ This study underscores the potential of the neoadjuvant setting for high-level correlative science, but also supports the need for biologically driven hypotheses and stratification of luminal subtypes, and also highlights the difficulties of serial analyses using high-dimensional data.

\section{Important genetic aberrations in ER-positive patients}

Some key genetic aberrations have been identified in recent years that can impact ER signaling and response to endocrine therapy. These aberrations may occur at the ER and other related surface receptor level (HER1, HER2, IGFR-1, FGFR-1), signaling pathway (PI3K, MAPK) level, and transcription factor level (myc, cyclin D1). It is important to note, that luminal A and B remains terminology for a phenotype, and that understanding the molecular aberration/s that drive the phenotype is likely to be critical for better outcomes. Following are some recent findings on the molecular alterations of key genes related to the ER pathway, their luminal phenotype and their potential clinical implications.

\section{HER2}

When ER binds to tamoxifen, it undergoes a conformational change that favors the recruitment of corepressors that inhibit transcriptional activity. ${ }^{57}$ The subset of ER-positive patients who are also HER2-positive are known to have a relatively poor response to tamoxifen. Patients whose tumors express high levels of both HER2 and the ER coactivator AIB1 (amplified in breast cancer 1) often develop tamoxifen resistance. ${ }^{58}$ Shou et al showed that addition of tamoxifen to the MCF-7/HER2-18 cell lines which overexpress both AIB1 and HER2 in fact resulted in growth stimulation. ${ }^{57}$

Ellis et al have reported that when neoadjuvant tamoxifen was administered, there was a significantly lower clinical response rate among patients with HER2 amplified tumors (33\%) as compared with the HER2 negative ones (49\%). ${ }^{59}$ They also reported that HER2 positivity was associated with a lower suppression of Ki67, a marker of cell proliferation, in response to tamoxifen. Hence, HER2+ is one driver of the luminal-B phenotype. Given its clinical and therapeutic implications, it should always be distinguished from other ER-positive cancers, including other luminal-B type cancers.

\section{PIK3CA}

PIK3CA mutations are seen in about $26 \%$ of breast cancers, especially in ER-positive and HER2-overexpressing subtypes (www.sanger.ac.uk/perl/genetics/CGP/cosmic). These mutations are frequently located in hotspots on exons 9 (helical domain) and 20 (kinase domain) ${ }^{60}$ The prognostic implications of this aberration are unclear, but recent data suggest it is likely that this mutation is associated with a relatively good prognosis for ER-positive, HER2-negative breast cancer. ${ }^{60-63}$ Although the exact mechanism for this is unclear, using gene 
expression and protein data from around 1800 breast tumor samples, we have reported that in ER-positive and HER2negative tumors, PIK3CA mutations were associated with relatively low mTORC1 signaling despite the presence of constitutive pathway activation, and with a good clinical outcome from single-agent tamoxifen. Increased sensitivity to tamoxifen has been also observed in PI3K mutant cell lines compared with wild-type, so this aberration may have important clinical implications.

PIK3CA and PIK3CB amplifications have been found in a minority of breast cancers. ${ }^{64}$ Amplification of this gene may have different functional effects compared with mutation for breast cancer. A more aggressive phenotype was reported with PIK3CA amplification compared with mutation in endometrial cancer. ${ }^{65}$

\section{AKTI}

AKT1 mutations have been found in around 4\% of breast cancers and have also been shown to be tumorigenic through activation of PI3K signaling. ${ }^{66}$ Whilst the association with tamoxifen and outcome is not known, AKT1 mutations were reported to have a gene expression profiles similar to those of PIK3CA mutants, suggesting that AKT1 mutations may also be associated with a relatively good clinical outcome from tamoxifen therapy. ${ }^{61}$ These hypotheses remain to be validated further in the clinical setting, and are highly relevant given the current intense interest in the development of PI3K pathway inhibitors.

\section{FGFR I}

Amplification of FGFR1 occurs in around 10\% of breast cancers, ${ }^{67,68}$ and amplification of FGFR1 has been associated with a poor prognosis in ER-positive breast cancer. ${ }^{69}$ Of note, HER2 and FGFR1 amplification rarely coexist, suggesting overlapping areas of pathway activation. FGFR1 amplification has been shown to be associated with the luminal B, ER-positive phenotype with high levels of proliferation, and hence may be a realistic potential therapeutic target for this poor prognostic group given that a number of drugs exist that inhibit this molecule. ${ }^{70}$ Additionally, FGFR1-amplified cell lines were shown to be resistant to tamoxifen, and this resistance was reversed by siRNA silencing of FGFR1. FGFR1-amplified tumors are frequently PR-negative.

\section{Myc and cyclin DI}

Musgrove et al have suggested that estrogen regulates cell growth principally via c-Myc. ${ }^{71}$ Overexpression of c-Myc and cyclin D1 occurs in 38\% (range 11\%-70\%) and 45\% (range $28 \%-81 \%$ ) of breast cancer patients and has been associated with tamoxifen resistance. ${ }^{72}$ Because these genes are involved with cell cycle regulation and proliferation activity, it is plausible that these aberrations also may be drivers of the luminal B phenotype.

\section{Limitations of current genomic predictors}

Despite their promise, none of aforementioned molecular aberrations, except for HER2, or gene signatures identified thus far has been approved for routine use in the clinical setting. There are a multitude of different reasons for this, from lack of appropriate clinical validation, unclear clinical implications, technical reasons involving standardization of the assay, tissue requirement (snap-frozen), and cost.

Although gene expression profiling has given us new insights into the biology of ER-positive breast cancer, a consistent definition of clinically relevant ER-positive subgroups is lacking. A widely accepted and standard definition could significantly help research in the field. Clinical trials could then be conducted in stratified populations because important therapy effects may be diluted or missed by analyzing an unselected ER-positive population. Whilst measurement of proliferation gene expression may provide a good starting point, the cutoff between luminal A and B groups is somewhat arbitrary due to the fact there is no clear biologic bimodality and that proliferation activity is a continuum. It will also be necessary in the future to include genetic aberrations as upfront stratification due to their differing influences on clinical outcome and response to therapy. Figure 1 demonstrates how our knowledge of the key genetic aberrations involved in the pathogenesis of the luminal subtypes has become a little clearer even in the last few years.

Whilst prognostic gene signatures have been consistently shown to be helpful in ER-positive breast cancers, they seem only to identify early relapses (within five years) and hence much of the biology underlying late relapse which is common in ER-positive breast cancer, and therefore prediction of it is unknown. We are also not sure to what extent gene predictors provide better prognostication than currently widely available clinicopathologic factors. A recent study reported that a four-marker surrogate immunohistochemical panel (ER, PR, HER2, and Ki67) was able to define a luminal B phenotype. This group, together with luminal/ HER2-positive tumors, was found to have worse relapsefree and breast cancer-specific survival compared with luminal A tumors, both in the presence and in the absence 

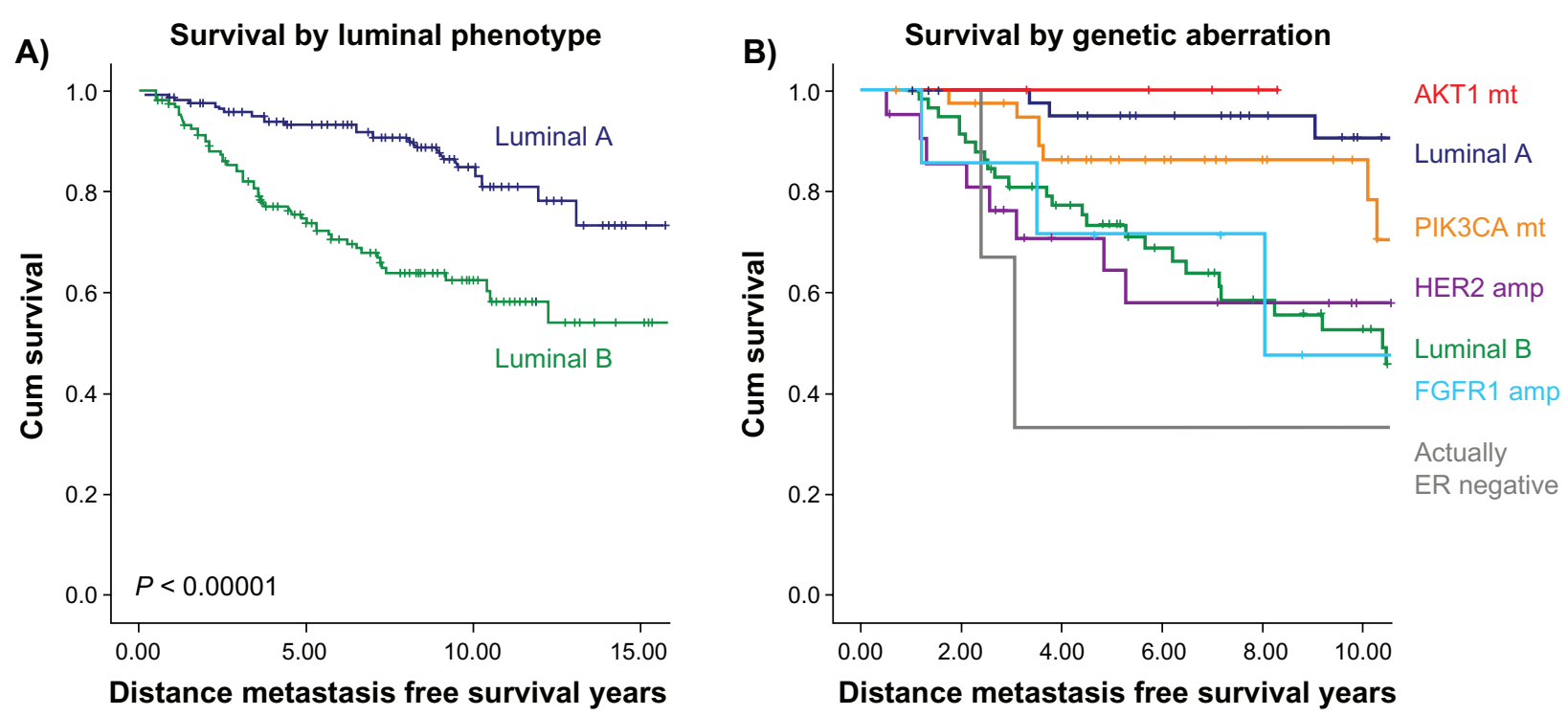

Figure I Kaplan-Meier graphs of different clinical outcomes of ER-positive breast cancer patients treated with adjuvant tamoxifen monotherapy. Patient series is described in reference 47. Previously the gene expression grade index (GGI) was used as a measure of proliferation activity to divide ER-positive breast cancer into luminal $A$ and $B$ phenotypes $\mathbf{A}$ ). Further limited knowledge of important DNA aberrations has identified PIK3CA and AKTI mutant breast cancers with a similar survival as the luminal $A$ subgroup and HER2 and FGFRI amplified breast cancers as the luminal B group. Hence, FGFRI represents a candidate molecular target for some luminal B tumors B). It is likely that both gene expression and molecular aberration may define a group of women suitable for tamoxifen monotherapy in the future.

of adjuvant systemic treatment with either tamoxifen alone or combination chemotherapy and tamoxifen. ${ }^{73}$ Notably for this study, all of these "standard" markers were performed in one central laboratory, emphasizing the poor reproducibility and quantification of local immunohistochemistry. The future may become clearer with the development of truly "predictive" biomarkers for tamoxifen response and resistance, and it is expected that a better understanding of the molecular drivers of ER-positive breast cancer using new deep and integrative genomic techniques will probably emerge. Furthermore, it has become apparent that it will be important to perform these correlative studies in the context of clinical trials to understand their clinical implications more accurately.

\section{Can we identify patients who will do well with tamoxifen alone?}

In general, the aforementioned studies that have evaluated the use of aromatase inhibitors versus tamoxifen have consistently shown that adding an aromatase inhibitor during adjuvant therapy improves disease-free survival and reduces the risk of breast cancer events compared with five years of tamoxifen monotherapy (see Table 1). However, if we look at absolute percentages, the reduction in risk of recurrence associated with aromatase inhibitors compared with tamoxifen could be considered disappointing. Upfront comparisons of tamoxifen and aromatase inhibitors are the same in terms of overall survival, supporting a main reduction from local and contralateral events (ie, not life-threatening) and even provocatively implying a worse outcome for aromatase inhibitor-treated patients after relapse and increased deaths from other causes (see Table 1). Two of the six trials of sequential treatment strategies yielded statistically significant improvements in overall survival compared with tamoxifen alone, although again the absolute difference in overall survival was small. Furthermore, aromatase inhibitors are not always well tolerated and are considerably more expensive than tamoxifen.

However, breast cancer events and early recurrence are important, and given that metastatic disease is incurable, the current standard is to include an aromatase inhibitor in the adjuvant treatment of postmenopausal women. ${ }^{74}$ Only a minority of postmenopausal women who decline or have a contraindication to an aromatase inhibitor currently receive tamoxifen alone. Because aromatase inhibitors do not suppress ovarian estrogen synthesis fully, they cannot be used for the treatment of premenopausal women. ${ }^{75}$

Recently, Viale et al developed a risk nomogram including tumor size, tumor grade, hormone receptor status, and HER2 status, to generate a risk model that could allow categorization of breast cancer patients into three groups, with low-, intermediate-, or high-risk outcomes. ${ }^{76}$ Low-risk patients achieved excellent outcomes regardless of the treatment type. However, as the risk of relapse increased, differences between the aromatase inhibitor-based treatments began to emerge. Therefore, there may still be a role for using five years of tamoxifen therapy for these lower-risk women. 
Research in the genomic field continues to advance our understanding of the biologic basis of relapse for ER-positive breast cancer, but clinical implementation of such findings remains slow. Currently it seems that the luminal A phenotype could do quite well with tamoxifen alone for five years. This subgroup could be identified in multiple ways, using either an immunohistochemical or gene expression assay that can quantify the expression of proliferation genes in a given breast cancer. Genetic aberrations, such as HER2 and FGFR1 amplification, could also define clearly for clinicians, without relying on arbitrary, nonbiologic cutoff for the luminal B phenotype, those ER-positive patients who will do poorly with tamoxifen treatment alone.

\section{Conclusion}

Tamoxifen treatment remains highly relevant for women diagnosed with ER-positive breast cancer. It is also clear that distinct luminal phenotypes exist and should be characterized by some proliferation marker in clinical trials, given that the treatment in the future is likely to be different for each. We feel that the most significant advances in the field of predictive biomarkers and biologic understanding will only occur in the context of innovative trials that incorporate prospectively planned translational research in their design. Hence, strong and respectful collaboration between basic researchers, clinicians, and pharmaceutical companies will be required in order to accomplish the challenging objective of a "personalized" treatment for ER-positive breast cancer patients.

\section{Disclosure}

The authors report no conflicts of interest in this work.

\section{References}

1. Tilghman SL, Nierth-Simpson EN, Wallace R, et al. Environmental hormones: Multiple pathways for response may lead to multiple disease outcomes. Steroids. 2010;75:520-523.

2. Nicholson RI, McClelland RA, Robertson JF, et al. Involvement of steroid hormone and growth factor cross-talk in endocrine response in breast cancer. Endocr Relat Cancer. 1999;6:373-387.

3. Pedram A, Razandi M, Levin ER. Nature of functional estrogen receptors at the plasma membrane. Mol Endocrinol. 2006;20:1996-2009.

4. Hammes SR, Levin ER. Extranuclear steroid receptors: Nature and actions. Endocr Rev. 2007;28:726-741.

5. Early Breast Cancer Trialists' Collaborative Group. Effects of chemotherapy and hormonal therapy for early breast cancer on recurrence and 15-year survival: An overview of the randomised trials. Lancet. 2005;365:1687-1717.

6. Osborne CK, Elledge RM, Fuqua SAW. Estrogen receptors in breast cancer therapy. Sci Med. 1996;3:32-41.

7. Arteaga CL, Osborne CK. Growth factors as mediators of estrogen/ antiestrogen action in human breast cancer cells. In: Lippman ME, Dickson RB, editors. Regulatory mechanisms in breast cancer: Advances in cellular and molecular biology of breast cancer. Boston, MA: Kluwer Academic; 1991.
8. Ellis PA, Saccani-Jotti G, Clarke R, et al. Induction of apoptosis by tamoxifen and ICI 182780 in primary breast cancer. Int J Cancer. 1997; 72:608-613.

9. Wiebe VJ, Osborne CK, Fuqua SAW, et al. Tamoxifen resistance in breast cancer. Crit Rev Oncol Hematol. 1993;14:173-188.

10. Horwitz KB, Jackson TA, Bain DL, et al. Nuclear receptor coactivators and corepressors. Mol Endocrinol. 1996;10:1167-1177.

11. Encarnacion CA, Ciocca DR, McGuire WL, et al. Measurement of steroid hormone receptors in breast cancer patients on tamoxifen. Breast Cancer Res Treat. 1993;26:237-246.

12. Hull DF III, Clark GM, Osborne CK, et al. Multiple estrogen receptor assays in human breast cancer. Cancer Res. 1983;43:413-416.

13. Boeddinghaus IM, Dowsett M. Comparative clinical pharmacology and pharmacokinetic interactions of aromatase inhibitors. J Steroid Biochem Mol Biol. 2001;79:85-91.

14. Buzdar A, Howell A. Advances in aromatase inhibition: Clinical efficacy and tolerability in the treatment of breast cancer. Clin Cancer Res. 2001;7:2620-2635.

15. Kao YC, Cam LL, Laughton CA, et al. Binding characteristics of seven inhibitors of human aromatase: A site-directed mutagenesis study. Cancer Res. 1996;56:3451-3460.

16. Johnston SR, Dowsett M. Aromatase inhibitors for breast cancer: Lessons from the laboratory. Nat Rev Cancer. 2003;3:821-831.

17. Jeng MH, Shupnik MA, Bender TP, et al. Estrogen receptor expression and function in long-term estrogen-deprived human breast cancer cells. Endocrinology. 1998;139:4164-4174.

18. Santen RJ, Song RX, Zhang Z, et al. Adaptive hypersensitivity to estrogen: Mechanisms and clinical relevance to aromatase inhibitor therapy in breast cancer treatment. J Steroid Biochem Mol Biol. 2005; 95:155-165.

19. Mouridsen H, Giobbie-Hurder A, Goldhirsch A, et al. Letrozole therapy alone or in sequence with tamoxifen in women with breast cancer. N Engl J Med. 2009;361:766-776.

20. Howell A, Robertson JF, Quaresma AJ, et al. Fulvestrant, formerly ICI 182,780 , is as effective as anastrozole in postmenopausal women with advanced breast cancer progressing after prior endocrine treatment. J Clin Oncol. 2002;20:3396-3403.

21. Osborne CK, Pippen J, Jones SE, et al. Double-blind, randomized trial comparing the efficacy and tolerability of fulvestrant versus anastrozole in postmenopausal women with advanced breast cancer progressing on prior endocrine therapy: Results of a North American trial. J Clin Oncol. 2002;20:3386-3395.

22. Howell A, Osborne CK, Morris C, et al. ICI 182,780 (Faslodex): Development of a novel, "pure" antiestrogen. Cancer. 2000;89:817-825.

23. Robertson JF, Nicholson RI, Bundred NJ, et al. Comparison of the short-term biological effects of 7alpha-[9-(4,4,5,5,5-pentafluoropentyl sulfinyl)-nonyl] estra-1,3,5,(10)-triene-3,17beta-diol (Faslodex) versus tamoxifen in postmenopausal women with primary breast cancer. Cancer Res. 2001;61:6739-6746.

24. Wakeling AE, Dukes M, Bowler J. A potent specific pure antiestrogen with clinical potential. Cancer Res. 1991;51:3867-3873.

25. Borras M, Laios I, el Khissiin A, et al. Estrogenic and antiestrogenic regulation of the half-life of covalently labeled estrogen receptor in MCF-7 breast cancer cells. J Steroid Biochem Mol Biol. 1996;57: 203-213.

26. Dauvois S, White R, Parker MG. The antiestrogen ICI 182780 disrupts estrogen receptor nucleocytoplasmic shuttling. J Cell Sci. 1993;106:1377-1388.

27. Howell A, Bergh J. Insights into the place of fulvestrant for the treatment of advanced endocrine responsive breast cancer. J Clin Oncol. 2010;28:4548-4550.

28. Coombes RC, Hall E, Gibson LJ, et al. A randomized trial of exemestane after two to three years of tamoxifen therapy in postmenopausal women with primary breast cancer. $N$ Engl J Med. 2004;350:1081-1092.

29. Howell A, Cuzick J, Baum M, et al. Results of the ATAC (Arimidex, Tamoxifen, Alone or in Combination) trial after completion of 5 years' adjuvant treatment for breast cancer. Lancet. 2005;365:60-62. 
30. Baum M, Budzar AU, Cuzick J, et al. Anastrozole alone or in combination with tamoxifen versus tamoxifen alone for adjuvant treatment of postmenopausal women with early breast cancer: First results of the ATAC randomised trial. Lancet. 2002;359:2131-2139.

31. Thurlimann B, Keshaviah A, Coates AS, et al. A comparison of letrozole and tamoxifen in postmenopausal women with early breast cancer. N Engl J Med. 2005;353:2747-2757.

32. Jones SE, Seynaeve C, Hasenburg A, et al. Results of the first planned analysis of the TEAM (tamoxifen exemestane adjuvant multinational) prospective randomized phase III trial in hormone sensitive postmenopausal early breast cancer. Abstr 15. 31st Annual San Antonio Breast Cancer Symposium, 2008 Dec 10-14; San Antonio, TX.

33. Rea D, Hasenburg A, Seynaeve C, et al. Five years of exemestane as initial therapy compared to 5 years of tamoxifen followed by exemestane: The TEAM trial, a prospective, randomized, phase III trial in postmenopausal women with hormone-sensitive early breast cancer. Abstr 11. 32nd Annual San Antonio Breast Cancer Symposium, 2009 Dec 9-13; San Antonio, TX.

34. Bliss JM, Kilburn LS, Coleman RE, et al. Disease related outcome with long term follow-up: An updated analysis of the Intergroup Exemestane Study (IES). Abstract 12. 32nd Annual San Antonio Breast Cancer Symposium, 2009 Dec 9-13; San Antonio, TX.

35. Jonat W, Gnant M, Boccardo F, et al. Effectiveness of switching from adjuvant tamoxifen to anastrozole in post-menopausal women with hormone-sensitive early-stage breast cancer: A meta-analysis. Lancet Oncol. 2006;7:991-996.

36. Goss PE, Ingle JN, Martino S, et al. Randomized trial of letrozole following tamoxifen as extended adjuvant therapy in receptor-positive breast cancer: Updated findings from NCIC CTG MA.17. J Natl Cancer Inst. 2005;97:1262-1271.

37. Goss PE, Ingle JN, Martino S, et al. A randomized trial of letrozole in postmenopausal women after five years of tamoxifen therapy for earlystage breast cancer. $N$ Engl J Med. 2003;349:1793-1802.

38. Goldhirsch A, Ingle JN, Gelber RD, et al. Thresholds for therapies: Highlights of the St Gallen international expert consensus on the primary therapy of early breast cancer 2009. Ann Oncol. 2009;20: 1319-1329.

39. Perou CM, Sorlie T, Eisen MB, et al. Molecular portraits of human breast tumours. Nature. 2000;406:747-752.

40. Sorlie T, Perou CM, Tibshirani R, et al. Gene expression patterns of breast carcinomas distinguish tumor subclasses with clinical implications. Proc Natl Acad Sci. 2001;98:10869-10874.

41. Sorlie T, Tibshirani R, Parker J, et al. Repeated observation of breast tumor subtypes in independent gene expression data sets. Proc Natl Acad Sci. 2003;100:8418-8423.

42. Sotiriou C, Neo SY, McShane LM, et al. Breast cancer classification and prognosis based on gene expression profiles from a population-based study. Proc Natl Acad Sci. 2003;1000:10393-10398.

43. Paik S, Shak S, Tang G, et al. A multigene assay to predict recurrence of tamoxifen treated node-negative breast cancer. $N$ Engl J Med. 2004;351:2817-2826

44. Ma XJ, Wang Z, Ryan PD, et al. A two-gene expression ratio predicts clinical outcome in breast cancer patients treated with tamoxifen. Cancer Cell. 2004;5:607-616.

45. Jansen MP, Foekens JA, van Staveren IL, et al. Molecular classification of tamoxifen resistant breast carcinomas by gene expression profiling. J Clin Oncol. 2005;23:732-740.

46. Oh DS, Troester MA, Usary J, et al. Estrogen-regulated genes predict survival in hormone receptor-positive breast cancers. J Clin Oncol. 2006;24:1656-1664.

47. Loi S, Haibe-Kains B, Desmedt C, et al. Definition of clinically distinct molecular subtypes in estrogen receptor positive breast carcinomas through genomic grade. J Clin Oncol. 2007;25:1239-1246.

48. Paik S, Shak S, Tang C, et al. Expression of the 21 genes in the recurrence score assay and tamoxifen clinical benefit in the NSABP study B-14 of node negative, estrogen receptor positive breast cancer. J Clin Oncol. 2005;23(16S):510.
49. Sotiriou C, Wirapati P, Loi S, et al. Gene expression profiling in breast cancer: Understanding the molecular basis of intermediate histologic grade to improve prognosis. J Natl Cancer Inst. 2006;98:1-12.

50. Paik S, Tang G, Shak S, et al. Gene expression and benefit of chemotherapy in women with node-negative, estrogen receptor-positive breast cancer. J Clin Oncol. 2006;24:3726-3734.

51. Albain KS, Barlow WE, Shak S, et al. Prognostic and predictive value of the 21-gene recurrence score assay in postmenopausal women with node-positive, oestrogen-receptor-positive breast cancer on chemotherapy: A retrospective analysis of a randomised trial. Lancet Oncol. 2010;11:55-65.

52. Symmans WF, Hatzis C, Sotiriou C, et al. Genomic index of sensitivity to endocrine therapy for breast cancer. J Clin Oncol. 2010;28: 4111-4119.

53. Chanrion M, Negre V, Fontaine $\mathrm{H}$, et al. A gene expression signature that can predict the recurrence of tamoxifen-treated primary breast cancer. Clin Cancer Res. 2008;14:1744-1752.

54. Miller WR, Larionov A, Renshaw L, et al. Gene expression profiles differentiating between breast cancer clinically responsive or resistant to letrozole. J Clin Oncol. 2009;27:1382-1387.

55. Mello-Grand M, Singh V, Ghimenti C, et al. Gene expression profiling and prediction of response to hormonal neoadjuvant treatment with anastrozole in surgically respectable breast cancer. Breast Cancer Res Treat. 2010;121:399-411.

56. Kok M, Linn S, Laar R. Comparison of gene expression profiles predicting progression in breast cancer patients treated with tamoxifen. Breast Cancer Res Treat. 2009;113:275-283.

57. Shou J, Massarweh S, Osborne CK, et al. Mechanisms of tamoxifen resistance: Increased estrogen receptor-HER2/neu cross-talk in ER/HER2-positive breast cancer. J Natl Cancer Inst. 2004;96: 926-935.

58. Osborne CK, Bardou V, Hopp TA, et al. Role of the estrogen receptor coactivator AIB1 (SRC-3) and HER-2/neu in tamoxifen resistance in breast cancer. J Natl Cancer Inst. 2003;95:353-361.

59. Ellis MJ, Tao Y, Young O, et al. Estrogen-independent proliferation is present in estrogen-receptor HER2-positive primary breast cancer after neoadjuvant letrozole. J Clin Oncol. 2006;24:3019-3025.

60. Stemke-Hale K, Gonzalez-Angulo AM, Lluch A, et al. An integrative genomic and proteomic analysis of PIK3CA, PTEN, and AKT mutations in breast cancer. Cancer Res. 2008;68:6084-6091.

61. Loi S, Haibe-Kains B, Majjaj S, et al. PIK3CA mutations associated with gene signature of low mTORC1 signaling and better outcomes in estrogen receptor-positive breast cancer. Proc Natl Acad Sci US A. 2010;107:10208-10213.

62. di Cosimo S, Baselga J. Phosphoinositide 3-kinase mutations in breast cancer: A "good" activating mutation? Clin Cancer Res. 2009;15: 5017-5019.

63. Kalinsky K, Jacks LM, Heguy A. PIK3CA mutation associates with improved outcome in breast cancer. Clin Cancer Res. 2009;15: 5049-5059.

64. Ellis MJ, Lin L, Crowder R. Phosphatidyl-inositol-3-kinase alpha catalytic subunit mutation and response to neoadjuvant endocrine therapy for estrogen receptor positive breast cancer. Breast Cancer Res Treat. 2010;119:379-390.

65. Salvesen HB, Carter SL, Mannelqvist M. Integrated genomic profiling of endometrial carcinoma associates aggressive tumors with indicators of PI3 kinase activation. Proc Natl Acad Sci U S A. 2009;106: $4834-4839$.

66. Carpten JD, Faber AL, Horn C. A transforming mutation in the pleckstrin homology domain of AKT1 in cancer. Nature. 2007;448: 439-444.

67. Courjal F, Cuny M, Simony-Lafontaine J, et al. Mapping of DNA amplifications at 15 chromosomal localizations in 1875 breast tumors: Definition of phenotypic groups. Cancer Res. 1997;57:4360-4367.

68. Theillet C, Adelaide J, Louason G, et al. FGFRI and PLAT genes and DNA amplification at 8p12 in breast and ovarian cancers. Genes Chromosomes Cancer. 1993;7:219-226. 
69. Elbauomy Elsheikh S, Green AR, Lambros MB. FGFR1 amplification in breast carcinomas: A chromogenic in situ hybridisation analysis. Breast Cancer Res. 2007;9:R23.

70. Turner N, Pearson A, Sharpe R, et al. FGFR1 amplification drives endocrine therapy resistance and is a therapeutic target in breast cancer. Cancer Res. 2010;70:2085-2094.

71. Musgrove EA, Sergio CM, Loi S, et al. Identification of functional networks of estrogen- and c-Myc-responsive genes and their relationship to response to tamoxifen therapy in breast cancer. PloS One. 2008;3: e2987.

72. Butt AJ, McNeil CM, Musgrove EA, et al. Downstream targets of growth factor and oestrogen signalling and endocrine resistance: The potential roles of c-Myc, cyclin D1 and cyclin E. Endocr Relat Cancer. 2005;12 Suppl 1:S47-S59.

73. Cheang M, Chia SK, Voduc D, et al. Ki67 index, HER2 status, and prognosis of patients with luminal B breast cancer. J Natl Cancer Inst. 2009;101:736-750.
74. Burstein HJ, Prestrud AA, Seidenfeld J. American Society of Clinical Oncology clinical practice guideline: Update on adjuvant endocrine therapy for women with hormone receptor-positive breast cancer. J Clin Oncol. 2010;28:3784-3796.

75. National Comprehensive Cancer Network. Clinical practice guidelines in oncology: Breast cancer. Available at: http:/www.nccn.org/ professionals/physician_gls/PDF/breast.pdf. Accessed 2010 May 25.

76. Viale G, Regan MM, Dell'Orto P, et al. Central review of ER, PgR and HER2 in BIG 1-98 evaluating letrozole vs letrozole followed by tamoxifen vs tamoxifen followed by letrozole as adjuvant endocrine therapy for postmenopausal women with hormone receptor-positive breast cancer. Abstract 76. 32nd Annual San Antonio Breast Cancer Symposium, 2009 Dec 9-13; San Antonio, TX.

\section{Publish your work in this journal}

OncoTargets and Therapy is an international, peer-reviewed, open access journal focusing on the pathological basis of all cancers, potential targets for therapy and treatment protocols employed to improve the management of cancer patients. The journal also focuses on the impact of management programs and new therapeutic agents and protocols on

\section{Dovepress}

patient perspectives such as quality of life, adherence and satisfaction The manuscript management system is completely online and includes a very quick and fair peer-review system, which is all easy to use. Visit http://www.dovepress.com/testimonials.php to read real quotes from published authors. 